\title{
Automated Identification of Paced Beats in Holter ECG
}

\author{
Filip Plesinger ${ }^{1}$, Ivo Viscor ${ }^{1}$, Radovan Smisek ${ }^{1}$, Josef Halamek ${ }^{1}$, Veronika Bulkova², Petr Nejedly ${ }^{1}$, \\ Adam Ivora $^{1}$, Magdalena Matejkova ${ }^{3}$, Pavel Leinveber ${ }^{3}$, Pavel Jurak ${ }^{1}$ \\ ${ }^{1}$ Institute of Scientific Instruments of the Czech Academy of Sciences, Brno, Czech Republic \\ ${ }^{2}$ Medical Data Transfer, Brno, Czech Republic \\ ${ }^{3}$ International Clinical Research Center at Saint Anne’s University Hospital, Brno, Czech Republic
}

\begin{abstract}
Background: Identification of paced beats is a necessity for Holter ECG monitoring reports. However, lower sampling frequencies make the pacing stimuli hard to identify. In this study, we present a method to distinguish between paced and non-paced beats.

Method: One-hour ECG recordings (158 patients, single lead, $250 \mathrm{~Hz}$ ) were recorded during usual daily activities. A total of 44,918 QRS complexes were detected and marked as paced $(19,004)$ or non-paced $(25,914)$. This dataset was split (60\%, out-of-patient) to training and testing datasets. Three features based on amplitude envelopes in two frequency bands were used to build a logistic regression model. An additional external dataset (2,193 recordings with 16,941 QRS) was assessed at a different facility and was used for the cross-database test.

Results: The model showed the test F1-score of 0.93, cross-database test shown F1-score of 0.924.

Conclusion: The presented method recognizes paced and non-paced heartbeats in lower sampling frequency, even if it can hardly be visually observed in the raw signal.
\end{abstract}

\section{Introduction}

Classification of beat types is a typical output of ECG analysis. And automated detection of pacemaker stimuli based on an analysis of analog or high-sampled digital signal (10-32 kHz) [1], [2] is the best option if technically allowed by acquisition hardware. However, widespread wearable ECG recorders (miniature holters, smartwatches) often record low-sampled signals (125-250-500 $\mathrm{Hz}$ ) due to the minimalization of consumed energy required for acquisition and wireless data transfer. Therefore, a single pacemaker stimulus, originally lasting $0.2-2 \mathrm{~ms}$, becomes wider and significantly smaller, as shown in fig. 1 in the case of $250 \mathrm{~Hz}$ data. A possible way to process low-sampled data may lead to an adaptive threshold [3] or a compressed sensing approach [4].
However, none of them was tested on ECG holter recordings acquired during usual daily activities, meaning that the ECG signal may contain noise caused by myopotentials or poor contact electrode contact.

Here, we present a method distinguishing between paced and non-paced beats, independently on pacing scenario (atriums, ventricles, combinations). The method is aimed at ECG signal acquired during regular daily activities.

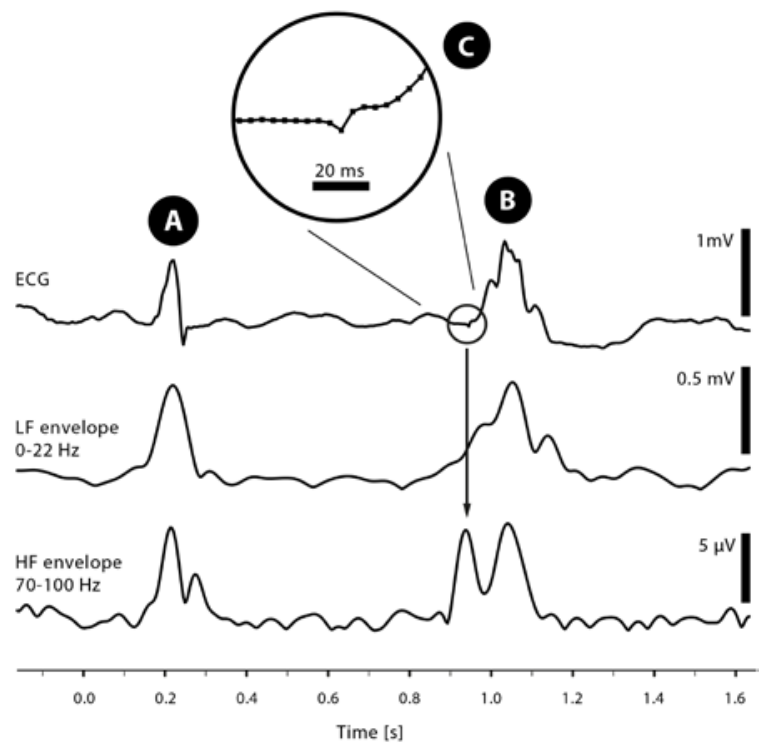

Figure 1. Raw Holter ECG signal (top) with non-paced (A) and paced (B) beat in data sampled at $250 \mathrm{~Hz}$ (Faros 180). The pacing stimulus (C, squares refer to samples) is barely visible in the raw signal. While the amplitude envelope LF $(0-22 \mathrm{~Hz}$ in the middle) mostly reflects QRS activity, the amplitude envelope $\mathrm{HF}(70-100 \mathrm{~Hz}$ at the bottom) can reveal pacing activity (arrow). 


\section{Data}

In this study, we used two independent datasets. Training and testing data (by MDT, s.r.o., Brno, Czechia) consisted of 1-lead, 198 Holter ECG recordings from 158 patients, each one hour long. Recordings (device Faros 180 by Bittium, Finland) were sampled at $250 \mathrm{~Hz}$ with 16-bit depth. Recordings were acquired during usual daily activities. QRS complexes ( $\mathrm{N}=44,918)$ were detected and marked as paced $(\mathrm{N}=19,004)$ or non-paced $(\mathrm{N}=25,914)$ in the SignalPlant software [5]. The dataset was split into training and testing sets in a $60 \%$ ratio $(\mathrm{N}=27,002$ and $\mathrm{N}=17,896)$. The training and testing part did not share patients, i.e., we prepared the out-of-patient test set.

We also used another dataset for independent, out-ofinstitution validation (by ICRC-FNUSA, Brno, Czechia). This dataset consists of 2,193 recordings from 494 patients, recorded in supine-resting position. Each record (device by M\&I, Prague, Czechia) was 10 seconds long, sampled at $5 \mathrm{kHz}$ with 24-bit depth. Data were filtered to $105 \mathrm{~Hz}$ and downsampled to $250 \mathrm{~Hz}$. QRS complexes in the ICRC-FNUSA dataset $(\mathrm{N}=16,941)$ were marked as paced $(\mathrm{N}=11,445)$ or non-paced $(\mathrm{N}=5,496)$ by presence and active-state of pacemaker during recording. While these recordings were 12-lead, we used only the Modified Chest Lead 5 (MCL5), since it was the most similar to the measuring setup of the MDT dataset.

\section{Method}

We present a solution based on the different behavior of paced and non-paced beats in specific frequencies of the ECG signal. These differences led us to the design of features and a classification model.

\subsection{Preprocessing}

While pacing artifacts are hardly visible in $250 \mathrm{~Hz}$ data (especially in Holter ECG contaminated by noise), signal transformations to amplitude envelopes in higher frequencies can reveal buried pacing artifacts. And such transformations in lower frequencies can, on the other hand, amplify QRS complexes and suppress any other activity in the signal. For these reasons, we extracted amplitude envelopes in two different bands: LF (0-22 Hz) to reveal QRS and HF (70-100 Hz) to reveal the pacing artifacts (Fig.1, Fig.2). Both envelopes were acquired with the Fast Fourier, and Hilbert transform with tapered cosine (i.e., Tukey) window with setting 0.5 .

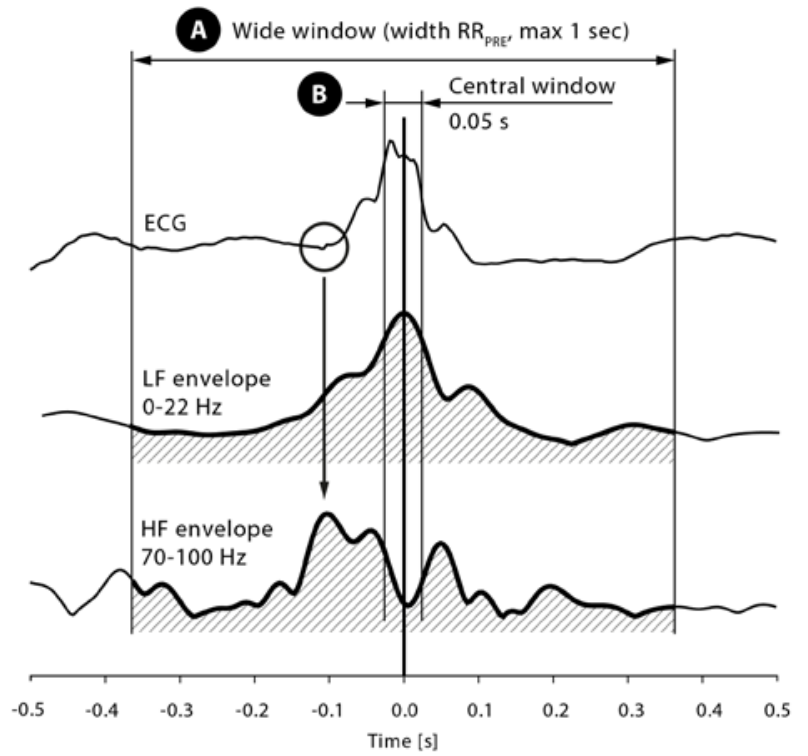

Figure 2. Raw ECG signal (top) with areas used for feature extraction from HF (middle) and LF (bottom) amplitude envelopes. "Wide window" (A) and "Central window” (B) refers to portions of the signal used for feature extraction. Windows are centered on the QRS annotation mark. RR refers to the delay between the preceding and actual QRS complex. Hatched areas indicate inputs for centers of gravity for the feature $\mathrm{G}_{\mathrm{HL}}$.

\subsection{Feature extraction for each QRS}

Features were extracted from the signal surrounding each QRS complex. The radius of the surrounding window was defined as half of the distance to previous QRS (RR) limited to $0.5 \mathrm{~s}$ (Fig.2A - the Wide window).

The first feature is based on a fact that if the pacing is present in a signal, its trace in the HF envelope creates maxima somewhere before QRS. And if the pacing is not present, then the HF envelope should be more similar to the LF because it contains only traces of QRS. Therefore, a high correlation between LF and HF should indicate a non-paced beat, while a low correlation between HF and LF should indicate pacing. This feature is computed as follows:

$$
C_{H L}=\operatorname{corr}(H F, L F)
$$

The second feature is also based on the same idea - we computed a temporal difference between centers of gravity of HF and LF envelopes (Fig.2, hatched areas). The difference approaching zero should indicate a non-paced beat because the highest activity should be very close in both envelopes. 
On the other hand, pacing preceding a QRS should move HF center of gravity before the QRS, and, therefore, this temporal difference will increase. This temporal difference of centers of gravity can be computed as

$$
G_{H L}=C_{o} G_{H}-C_{o} G_{L}
$$

where $\mathrm{CoG}_{\mathrm{H}}$ or $\mathrm{Cog}_{\mathrm{L}}$ are centers of gravity in seconds from $\mathrm{HF}$ and LF envelopes, respectively.

The last feature examines only the HF envelope. It compares HF maximum from the wide surrounding window (QRS $\pm \mathrm{RR} / 2$, Fig.2A) to HF maximum from a central window (QRS $\pm 0.025 \mathrm{~s}$, Fig.2B). If pacing is present, then HF will contain a pacing trace possibly stronger then QRS trace and, therefore, this ratio will be higher then one. If the pacing is not present, then overall maxima will stay very close to the QRS, and this ratio will be one. This third feature is computed as

$$
R_{H C}=\frac{\max \left(H F_{\text {Wide }}\right)}{\max \left(H F_{\text {Central }}\right)}
$$

Presented features were extracted for all QRS complexes from the MDT and ICRC-FNUSA datasets, and their ability to distinguish between paced and the nonpaced beat was measured as the area under the receiveroperator curve (AUC). Features were checked for a linear relationship using Pearson correlation (MDT only).

\subsection{Logistic regression model}

Features from a training part of the MDT dataset were z-score standardized and entered a logistic regression model. Finally, the model was tested using the testing part of the MDT dataset and also by the independent ICRCFNUSA dataset.

\section{4. $\quad$ Results}

Features AUC was measured using all MDT and ICRCFNUSA data due to a low number of patients. The ability to separate paced and non-paced beats in both datasets is shown in Fig. 3 and 4. Correlations between features are shown in Tab 1.

Table 1. Mutual correlations between features (MDT)

\begin{tabular}{lccc}
\hline Feature & $\mathrm{C}_{\mathrm{HL}}$ & $\mathrm{G}_{\mathrm{HL}}$ & $\mathrm{R}_{\mathrm{HC}}$ \\
\hline $\mathrm{C}_{\mathrm{HL}}$ & 1 & 0.531 & -0.292 \\
$\mathrm{G}_{\mathrm{HL}}$ & 0.531 & 1 & -0.203 \\
$\mathrm{R}_{\mathrm{HC}}$ & -0.292 & -0.203 & 1 \\
\hline
\end{tabular}

Values required for standardization (mean \pm std) were extracted from the training set only (Tab. 2). These values were later used for the standardization of testing data.
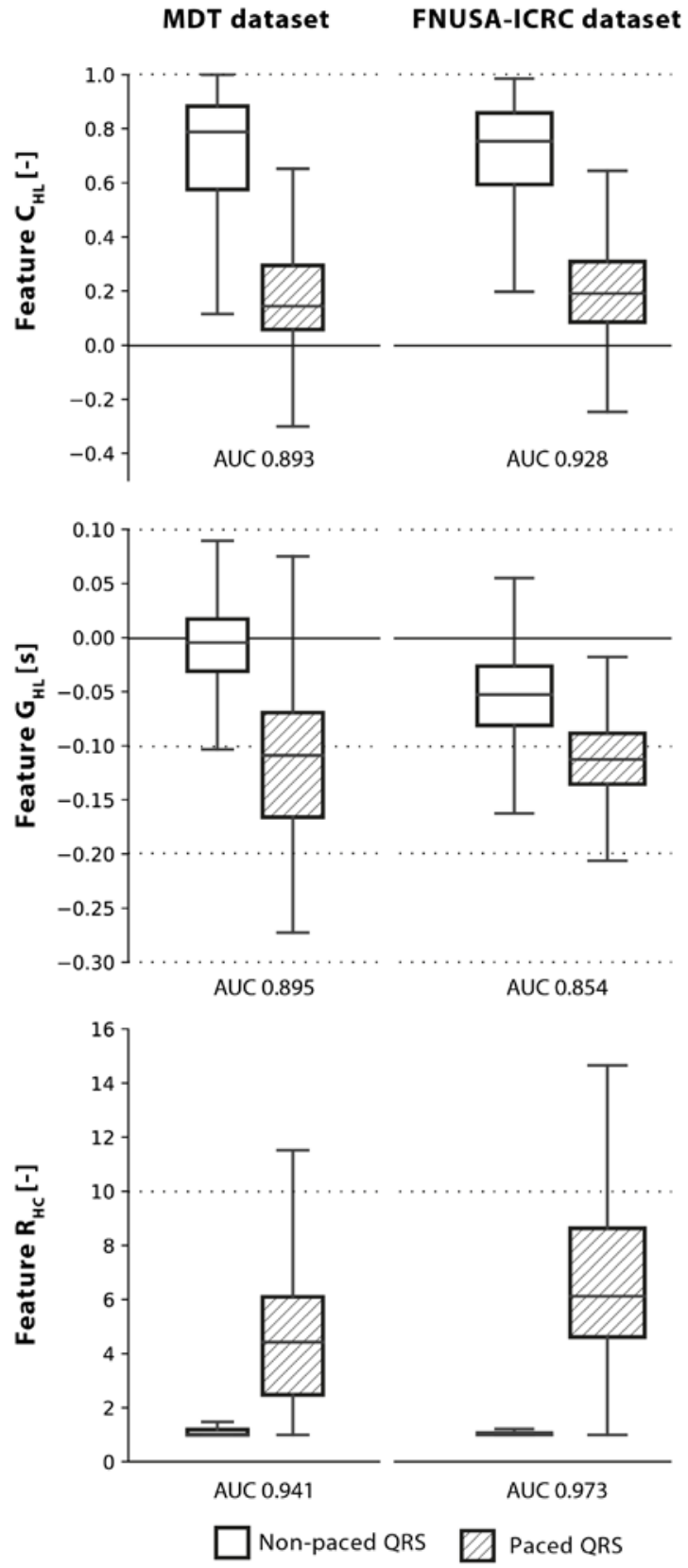

Figure 3. Features (rows) in the MDT data (left) and ICRCFNUSA data (right). The AUC values represent the ability of features to separate paced (hatched) and non-paced (clear) beats.

Table 2. Standardization values for features entering a model. Values are based on the MDT training dataset

\begin{tabular}{lcc}
\hline Feature & Mean & $\begin{array}{c}\text { Standard } \\
\text { deviation }\end{array}$ \\
\hline $\mathrm{C}_{\mathrm{HL}}$ & 0.4735 & 0.4735 \\
$\mathrm{G}_{\mathrm{HL}}$ & -0.0457 & 0.0702 \\
$\mathrm{R}_{\mathrm{HC}}$ & 2.6509 & 6.1192 \\
\hline
\end{tabular}




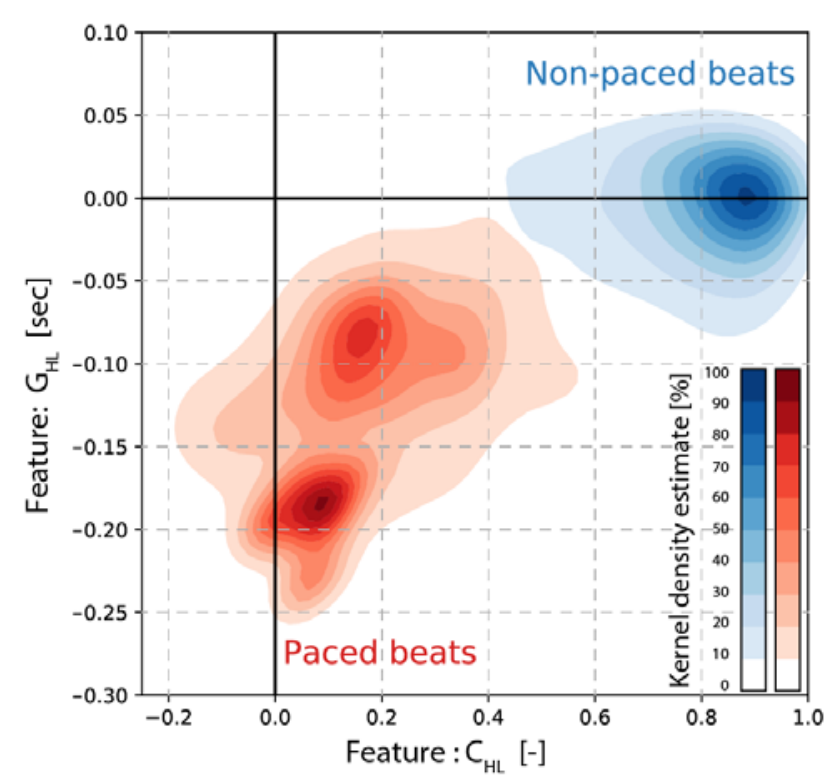

Figure 4. The relation between $\mathrm{C}_{\mathrm{HL}}$ and $\mathrm{G}_{\mathrm{HL}}$ features for paced (red) and non-paced (blue) heartbeats in the MDT dataset. Peaks in the paced-beats area might refer to different kinds of stimulation.

Next, a logistic regression model (Tab.3) was trained using the standardized MDT training set and Statsmodels [6] Python package. Training lasts 12 iterations with the LBFGS solver. Feature significance was checked with Ztest.

Table 3. The logistic regression model

\begin{tabular}{lcc}
\hline Feature & Coefficient & p-value \\
\hline $\mathrm{C}_{\mathrm{HL}}$ & 0.7911 & $<0.001$ \\
$\mathrm{G}_{\mathrm{HL}}$ & 1.7401 & $<0.001$ \\
$\mathrm{R}_{\mathrm{HC}}$ & -5.5235 & $<0.001$ \\
Intercept & -0.1213 & $<0.001$ \\
\hline
\end{tabular}

Finally, the trained model was evaluated using the MDT testing set and ICRC-FNUSA dataset (Tab.4). The MDT test data showed 1,194 cases of misclassification in 17,896 recordings with 461 cases of non-recognized pacing. False indication of pacing (766 cases) consisted of 408, 82, and 276 cases of sinus beats, premature ventricular contraction, and premature atrial contractions, respectively.

Table 4. Model results for different datasets

\begin{tabular}{lcccc}
\hline Dataset & QRS & Accuracy & $\begin{array}{c}\text { F1 } \\
\text { (micro) }\end{array}$ & $\begin{array}{c}\text { F1 } \\
\text { (macro) }\end{array}$ \\
\hline MDT-train & 27,002 & 0.911 & 0.911 & 0.908 \\
MDT-test & 17,896 & 0.931 & 0.931 & 0.931 \\
ICRC- & 16,941 & 0.933 & 0.933 & 0.924 \\
FNUSA & & & & \\
\hline
\end{tabular}

\section{Discussion and Conclusion}

We presented a logistic regression model to distinguish whether a heartbeat was paced or not. Relations between F1 scores from tests on MDT and independent ICRCFNUSA datasets suggest a high generalization ability of extracted features and the resultant model. However, analysis of misclassified beats (7.3\%) showed that, for example, intense noise could affect the HF envelope at such a level that the $\mathrm{C}_{\mathrm{HL}}$ will drop down to zero (compare with feature behavior in Fig.3 and Fig.4). Contrary to existing approaches, this was done in $250 \mathrm{~Hz}$ Holter ECG data acquired during the usual daily activities of patients (in the case of the MDT dataset). Further studies should focus on increasing robustness against noise.

\section{Acknowledgments}

The research was supported by a research grant no. FW01010305 by the Czech Technological Agency and by the Czech Academy of Sciences (project RVO:68081731).

\section{References}

[1] M. Jennings, B. Devine, S. Luo, and P. W. Macfarlane, "Enhanced software based detection of implanted cardiac pacemaker stimuli," in Computers in Cardiology, 2009.

[2] A. Polpetta and P. Banelli, "Fully digital pacemaker detection in ECG signals using a nonlinear filtering approach," in Proceedings of the 30th Annual International Conference of the IEEE Engineering in Medicine and Biology Society, EMBS'08 - "Personalized Healthcare through Technology," 2008.

[3] E. D. Helfenbein, J. M. Lindauer, S. H. Zhou, R. E. Gregg, and E. C. Herleikson, "A software-based pacemaker pulse detection and paced rhythm classification algorithm," in Journal of Electrocardiology, 2002.

[4] M. Shoaib and H. Garudadri, "Digital pacer detection in diagnostic grade ECG," in 2011 IEEE 13th International Conference on e-Health Networking, Applications and Services, HEALTHCOM 2011, 2011.

[5] F. Plesinger, J. Jurco, J. Halamek, and P. Jurak, "SignalPlant: an open signal processing software platform,” Physiol. Meas., vol. 37, no. 7, pp. N38N48, 2016.

[6] S. Seabold and J. Perktold, "Statsmodels: Econometric and Statistical Modeling with Python,” Proc. 9th Python Sci. Conf, 2010.

Address for correspondence:

Filip Plesinger, Kralovopolska 147, Brno, Czech Republic

Email: fplesinger@isibrno.cz 